\title{
Directed Radiation for Far-Field Wireless Power Transfer
}

\author{
Mutasem Alsharqi \\ M.Sc \\ Electrical Engineering Department, \\ Alumnus \\ California State University, Fullerton \\ 800 N. State College Blvd \\ Fullerton CA, 92831
}

\author{
Mohammed Hilfi \\ M.Sc \\ Electrical Engineering Department, \\ Lecturer \\ California State University, Fullerton \\ 800 N. State College Blvd \\ Fullerton CA, 92831
}

\author{
David Cheng, PhD \\ Electrical Engineering Department, \\ Professor \\ California State University, Fullerton \\ 800 N. State College Blvd \\ Fullerton CA, 92831
}

\begin{abstract}
Wireless Power Transfer (WPT) is a field of science where energy can be transferred between two points (such as from a power source to an electrical load or consuming device) to charge electronic devices wirelessly. Examples of such electronic devices are cellphones, portable computers (laptops), integrated circuits, light emitting diodes, and electric vehicles. WPT can avoid the disadvantages of the traditional charging systems such as, power failure due short circuits, and dangerous battery materials. The advantages of WPT in comparison to the wired system include reducing the copper material, which is necessary to make cables, transmit power to isolated areas (deserts, mountains, and islands) where wired transmission is very difficult to implement, and the reduction of expenses. Wireless transmission is beneficial in cases where using cables or attached wires can be dangerous or unavailable. This research paper focuses on finding a valid distance range for Friis' equation. MATLAB ${ }^{\circledR}$ program was used to calculate the predicted results for farfield WPT. By looking at the results, it is safe to conclude that the directed WPT is much more convenient in terms of transmitting covered distance and the Beam Efficiency compared to the isotropic WPT.
\end{abstract}

\section{General Terms}

Wireless Power Transfer (WPT)

\section{Keywords}

Wireless Power Transfer (WPT), Friis' equation, Far-Field WPT, Beam Efficiency, Wireless Electricity (WiTricity)

\section{INTRODUCTION}

Wireless Power Transfer (WPT) bases use electromagnetic field, is based on the radio-waves theory. WPT is a term that involves several different technologies for transmitting energy in electromagnetic fields. The transmitter converts the input power to a time-varying electromagnetic field. The receiver receives the power and converts it back to DC or AC electric current, which would be suitable to use in the electric load. This electrical load may be mobile, laptop, or even hybrid electric vehicle. The type of antenna in the WPT may vary according to the field it makes. This field could be an electric field if the antenna is a metal plate, a magnetic field if the antenna is coil, a microwave antenna that radiates radiowaves, or a laser antenna that radiates light. At the receiver's side, a similar antenna (Rectenna) returns the oscillating field to an electric current.

\section{PROBLEM STATEMENT}

Traditional charging systems have many disadvantages, such as the continuous need to plug electronic devices to a power outlet at home or to plug it into a power outlet in a car. This means the stored power amount in these electronic devices is proportional to the plugging time. Thus, in many cases users find themselves out of charge and want to go home or to any power stand just to charge their portable devices.

\section{CONTRIBUTIONS AND OBJECTIVES}

Imagine that if the mobile phones, Portable Computers (PCs), implanted medical devices, robots, street Light Emitting Diodes (LED) lights, Integrated circuits (ICs), and even Electrical Vehicles (EVs) could be powered without wires, or batteries $[1,2]$. Figure 1 shows an imaginary room without wires (Wireless Electricity (WiTricity) charger).

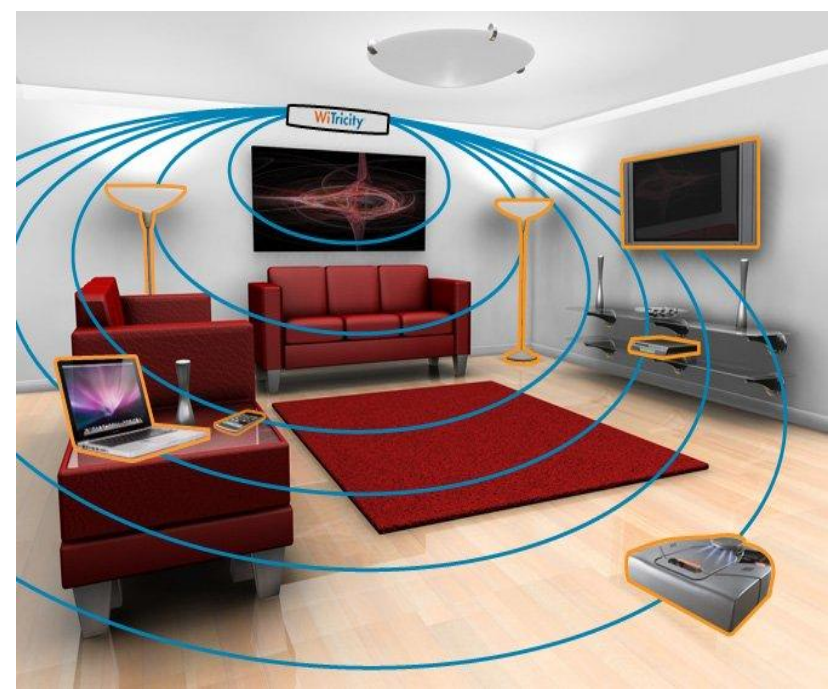

Figure 1: Wireless Electricity (WiTricity) charger [1]

\section{WPT EXPERIMENTS}

Tesla in 1899 built a huge coil connected to a high tower with a height of $200 \mathrm{ft}$. and a ball diameter of $3 \mathrm{ft}$. [3]. This was known as "Tesla Tower". The coil was fed by Tesla with 300 $\mathrm{kW}$ of power and had a resonated frequency of $150 \mathrm{kHz}$; in addition, at the top sphere he reached $100 \mathrm{MV}$ [4]. Unfortunately, this experiment failed due to the widespread transmitted power in all directions because the $150 \mathrm{kHz}$ frequency made almost $2000 \mathrm{~m}$ wavelength $\left(\lambda=\frac{c}{f}\right)[4]$, and a huge transmitting antenna size [5]. Here the sphere ball on top of Tesla tower acts like an isotropic source which tansmitts energy in all directions; it is not directed to a spcific location or receiver.

Many developments showed that WPT was possible in late 1950 's. Fundamentals and practical experiments results collected by Schwering and Goubau confirmed that 
transmitting the microwave power with approximately $100 \%$ efficiency is likely to happen using reflecting mirrors or lenses to guide a wave beam [6]. This result is extremely important because it dispelled the assumption that power density will always descend by the inverse square law (the square of the distance) $[3,6]$.

William C. Brown made an interesting experiment in October 1964 to launch a helicopter with 60 feet above the transmitting antenna using Microwave Power Transfer (MPT) system. Brown used $3 \mathrm{GHZ}$ microwave frequency [3, 4]. In November of the same year, a 10 hour sustained flight was achieved [3].

\section{FRISS EQUATION}

All electromagnetic waves are energy. Thus, hypothetically, all electromagnetic waves could be used for WPT [4]. Friss equation used to calculate the receiving power at a far-field (radiative) distance within sufficient far-field conditions for plane waves as follows [4, 7]:

$\operatorname{Pr}=\frac{\lambda 2 \mathrm{GtGr}}{(4 \pi D) 2} P t=\frac{\mathrm{At} \mathrm{Ar}}{(\lambda D) 2} P t$

Where Pr: the received power in (W), $\lambda$ : the wavelength in (m), Gt: the antenna gain of the transmitting antenna (unitless), Gr: the antenna gain of the receiving antenna (unit-less), $\mathrm{D}$ : the distance between the transmitting antenna and the receiving antenna in $(\mathrm{m})$, At: the aperture area of the transmitting antenna in (m2), Ar: the aperture area of the receiving antenna in $(\mathrm{m} 2), \mathrm{Pt}$ : the transmitted power. If the distance between the transmitter and the receiver is too short; Friis's equation is invalid because $\tau 2$ becomes extremely large, which means the plane wave at the receiving antenna plane is unavailable[4].

To compute the receiving power or the Beam Efficiency (BE), is denoted as $\eta$ (ETA, pronounced [ê:ta:]), $\tau$ parameter is available;

$\lambda^{2}=\frac{A_{t} A_{r}}{(\lambda D)^{2}}$

$\eta=\frac{\mathrm{P}_{\mathrm{r}}}{\mathrm{P}_{\mathrm{t}}}=1-e^{-\left[\frac{A t A r}{(\lambda D)^{2}}\right]}=1-e^{-\tau^{2}}$

\section{ISOTROPIC ((UNDIRECTED) RADIATION PROBLEM}

The current study made an estimation to understand what happened with Tesla using the Friis equation. The following is the Friis equation estimation:

Assuming the refractive index $\mathrm{n} \cong 1$ for air, where $\mathrm{n}=\mathrm{c} / \mathrm{v}$. The symbol (c) represents the speed of light in free space (vacuum), $c=3 * 108 \mathrm{~m} / \mathrm{s}$. Where $\mathrm{v}$ : the speed of light in the desired medium (in this situation the desired medium is air). So, transmitting power in air will be approximately the same as transmitting power in free space (vacuum), for this reason c $\cong \mathrm{v}$. Using the same frequency (Tesla experiment frequency) $\mathrm{f}=150 \mathrm{kHz}$, then the wavelength $\lambda=\mathrm{v} / \mathrm{f}=\mathrm{c} / \mathrm{f}, \lambda=3^{*} 108 /$ $150,000=2000 \mathrm{~m}$. Assuming the aperture area of the transmitting antenna At $=60.96 \mathrm{~m} 2$; it is proportional to the tower length $(200 \mathrm{ft}=60.96 \mathrm{~m})$, and $\left(\mathrm{At} \leq \frac{1}{2} \lambda \leftrightarrow 60.96 \leq\right.$ $\left.\left(\frac{1}{2} * 2000=1000\right)\right)$. Also, assuming the aperture area of the receiving antenna $\mathrm{Ar}=60.96 \mathrm{~m} 2\left(\mathrm{Ar} \leq \frac{1}{2} \lambda\right)$. Using the same transmitted power Tesla used, $\mathrm{Pt}=300 \mathrm{~kW}=(3 * 105) \mathrm{W}$. Then at any distance, it is possible to calculate the received power in (W) using Friis equation;
$\operatorname{Pr}=\frac{\lambda 2 \mathrm{Gt} \mathrm{Gr}}{(4 \pi \mathrm{D}) 2} \mathrm{Pt}=\frac{\mathrm{At} \mathrm{Ar}}{(\lambda \mathrm{D}) 2} \mathrm{Pt}$, as following:

At 10 meters from the antenna, $\operatorname{Pr}=\frac{60.96 * 60.96}{(2000 * 10) 2} *(3 * 105)=$ $2.787 \mathrm{~W}$.

At 100 meters from the antenna, $\operatorname{Pr}=\frac{60.96 * 60.96}{(2000 * 100) 2} *(3 * 105)=$ $2.787 * 10-2 \mathrm{~W}$.

At 1000 meters from the antenna, $\operatorname{Pr}=\frac{60.96 * 60.96}{(2000 * 1000) 2} *(3 *$ $105)=2.787 * 10-4 \mathrm{~W}$.

For additional distances, the same equation should be used. From previous results, it is clear that a very small fraction of the transmitted power was received.

\section{DIRECTED RADIATION}

There are many methods to increase the radiated power and the beam efficiency, such as using a higher frequency, using a directed antenna towards a target, and the previous methods combined. All methods are discussed in the following subsections:

\section{1) Using A Higher Frequency}

Using Brown's experiment frequency $\mathrm{f}=3 \mathrm{GHz}$, then the wavelength $\lambda=\mathrm{v} / \mathrm{f}=\mathrm{c} / \mathrm{f}, \lambda=3 * 108 / 3 * 109=0.1 \mathrm{~m}$. The wavelength decreases from $2000 \mathrm{~m}$ to $0.1 \mathrm{~m}$. While assuming At $=0.025 \mathrm{~m} 2$ and $\mathrm{Ar}=0.025 \mathrm{~m} 2\left(\mathrm{At} \leq \frac{1}{2} \lambda, \mathrm{Ar} \leq \frac{1}{2} \lambda\right)$. Using the same transmitted power Tesla used in his experiment, $\mathrm{Pt}=$ $300 \mathrm{~kW}=(3 * 105) \mathrm{W}$. Then at any distance, the received power:

At 10 meters from the antenna, $\operatorname{Pr}=\frac{0.025 * 0.025}{(0.1 * 10) 2} *(3 * 105)=$ $187 \mathrm{~W}$.

At 100 meters from the antenna, $\operatorname{Pr}=\frac{0.025 * 0.025}{(0.1 * 100) 2} *(3 * 105)=$ $1.87 \mathrm{~W}$.

At 1000 meters from the antenna, $\operatorname{Pr}=\frac{0.025 * 0.025}{(0.1 * 1000) 2} *(3 * 105)$ $=1.87 * 10-2 \mathrm{~W}$.

\section{2) Using Directed Antenna}

Figure 2 shows antenna gain for different volumes, in other words, it shows the difference between the isotropic radiation and radiation in specific volumes (hemisphere, quarter sphere, and segment). In Figure 2, PD is the power density. The number 2 in Figure 2 (b) represents the ratio between the full sphere volume and the hemisphere volume (full sphere volume $=2 *$ hemisphere volume). The number 4 in Figure 2 (c) represents the ratio between the full sphere volume and the quarter sphere volume (full sphere volume $=4 *$ quarter sphere volume). The number 18334 in Figure 2 (d) represents the ratio between the full sphere volume and the segment volume (full sphere volume $=18334 *$ segment volume). 


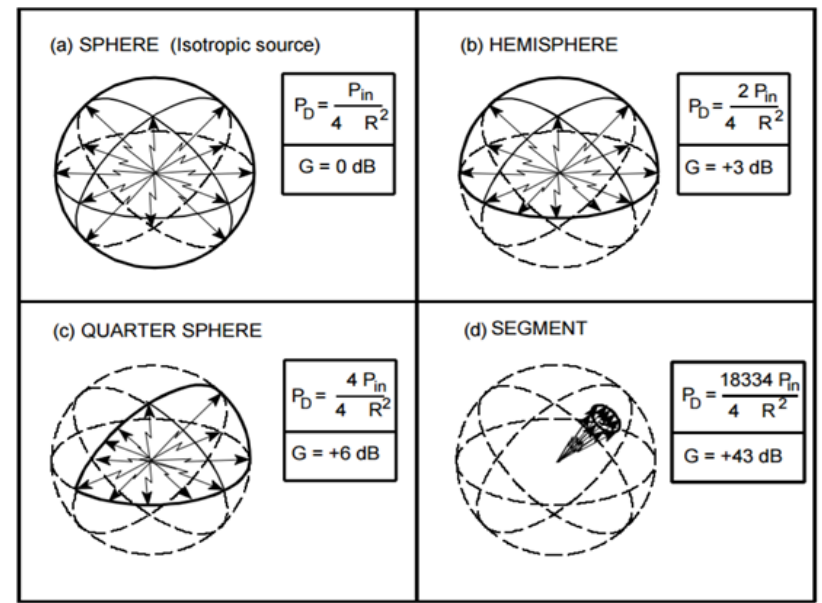

Figure 2: Antenna gain for different volumes: (a) isotropic (full sphere), (b) hemisphere, (c)quarter sphere, and (d) segment [8]

Using the same frequency (Tesla experiment frequency) $f=$ $150 \mathrm{kHz}$, then $\lambda=2000 \mathrm{~m}$. Also, keeping the At $=60.96 \mathrm{~m} 2$ and $\mathrm{Ar}=60.96 \mathrm{~m} 2$. Using the same transmitted power Tesla used in his experiment, $\mathrm{Pt}=300 \mathrm{~kW}=(3 * 105) \mathrm{W}$. Now, the whole equation is multiplied by the new constant, the volume constant $=18334$.

At 10 meters from the antenna, $\operatorname{Pr}=\frac{60.96 * 60.96}{(2000 * 10) 2} *(3 * 105) *$ $18334=5.109 * 104 \mathrm{~W}$.

At 100 meters from the antenna, $\operatorname{Pr}=\frac{60.96 * 60.96}{(2000 * 100) 2} *(3 * 105) *$ $18334=510.9 \mathrm{~W}$.

At 1000 meters from the antenna, $\operatorname{Pr}=\frac{60.96 * 60.96}{(2000 * 1000) 2} *(3 *$ $105) * 18334=5.019 \mathrm{~W}$

\section{3) Using Higher Frequency And Directed Antenna Together}

Using Brown's experiment frequency $\mathrm{f}=3 \mathrm{GHz}$, then $\lambda=0.1$ $\mathrm{m}$. Keeping the At $=0.025 \mathrm{~m} 2$ and $\mathrm{Ar}=0.025 \mathrm{~m} 2$. Using the same transmitted power Tesla used in his experiment, $\mathrm{Pt}=$ $300 \mathrm{~kW}=(3 * 105) \mathrm{W}$. Now, the whole equation is multiplied by the new constant, the volume constant $=18334$.

At 10 meters from the antenna, $\operatorname{Pr}=\frac{0.025 * 0.025}{(0.1 * 10) 2} *(3 * 105) *$ $18334=3.437 * 106 \mathrm{~W}$ (Invalid, will be discussed later in Friis equation valid distance range).

At 100 meters from the antenna, $\operatorname{Pr}=\frac{0.025 * 0.025}{(0.1 * 100) 2} *(3 * 105) *$ $18334=3.437 * 104 \mathrm{~W}$

At 1000 meters from the antenna, $\operatorname{Pr}=\frac{15 * 1}{(0.1 * 1000) 2} *(3 * 105)$ * $18334=343$

\section{FRIIS EQUATION VALID DISTANCE RANGE}

The invalid results are not acceptable because Pr > Pt. To solve this issue, it is necessary to find the valid distance range (minimum and maximum distances from the transmitter) for the Friis equation. To find the minimum distance, the current study developed a new model, which is shown below:

$\operatorname{Pr} \leq\left(\frac{\text { At Ar }}{(\lambda \mathrm{D})^{2}} \mathrm{Pt} *\right.$ Volume constant $)$
By assuming the minimum value of $\mathrm{Pr}$ is $1 \mathrm{~W}$ and is always less than the right side in equation (4) yields:

$1 \leq\left(\frac{\mathrm{At} \mathrm{Ar}}{(\lambda \mathrm{D})^{2}} *\right.$ Volume constant $)=\frac{1}{1} \leq$

$\left(\frac{\text { At Ar }}{(\lambda \mathrm{D})^{2}} *\right.$ Volume constant $)$

By doing cross product yields:

$(\lambda \mathrm{D})^{2} \geq \mathrm{At} \mathrm{Ar} *$ Volume constant

$\lambda 2 \mathrm{D} 2 \geq$ At Ar $*$ Volume constant

By dividing each side of equation (7) with $\frac{1}{\lambda^{2}}$ yields:

$\mathrm{D} 2 \geq \frac{\mathrm{At} \mathrm{Ar}}{\lambda^{2}} *$ Volume constant

By taking the square root for each side of equation (8) yields:

$\mathrm{D} \geq+\sqrt[2]{\frac{\mathrm{At} \mathrm{Ar}}{\lambda^{2}} * \text { Volume constant }}$

The last equation (9) represents the minimum distance acceptable to start receiving energy for the Friis equation.

To find the maximum distance that the energy could reach from the transmitter antenna using the Friis equation, with the assumption that the minimum accepting energy at that distance is $1 \mathrm{~W}$, this research paper developed a new model which is shown below:

$\left(\frac{\mathrm{At} \mathrm{Ar}}{(\lambda \mathrm{D})^{2}} \mathrm{Pt} *\right.$ Volume constant $) \geq 1 \mathrm{~W}$

$\left(\frac{\text { At Ar }}{(\lambda \mathrm{D})^{2}} \mathrm{Pt} *\right.$ Volume constant $) \geq \frac{1}{1}$

By doing cross product yields:

$(\lambda \mathrm{D})^{2} \leq \mathrm{At} \mathrm{Ar} * \mathrm{Pt} *$ Volume constant

$\lambda 2 \mathrm{D} 2 \leq \mathrm{At} \mathrm{Ar} * \mathrm{Pt} *$ Volume constant

By dividing each side of equation (13) with $\frac{1}{\lambda^{2}}$ yields:

$\mathrm{D} 2 \leq \frac{\text { At Ar }}{\lambda^{2}} * \mathrm{Pt} *$ Volume constant

By taking the square root for each side of equation (14) yields:

$\mathrm{D} \leq+\sqrt[2]{\frac{\mathrm{At} \mathrm{Ar}}{\lambda^{2}} * \mathrm{Pt} * \text { Volume constant }}$

The last equation (15) represents the maximum distance acceptable to receive energy for the Friis equation.

To find the maximum distance that the energy could reach from the transmitter antenna using the Friis equation, with the assumption that the minimum accepting energy at that distance is any desired watts (x W), divide the denominator of equation (15) with that desired receiving energy, this research paper developed a new model which is presented below:

$\mathrm{D} \leq+\sqrt[2]{\frac{\mathrm{At} \mathrm{Ar}}{\lambda^{2} * \mathrm{X}} * \mathrm{Pt} * \text { Volume constant }}$

\section{APPLYING FRIIS EQUATION VALID DISTANCE RANGE}

To find the minimum distance (nearest distance from the transmitter) for the higher frequency part, equation (9) should be applied as follows:

$\mathrm{D} \geq+\sqrt[2]{\frac{\mathrm{At} \mathrm{Ar}}{\lambda^{2}} * \text { Volume constant }} \leftrightarrow \mathrm{D} \geq+\sqrt[2]{\frac{0.025 * 0.025}{(0.1)^{2}} * 1}$ 
So, $\mathrm{D} \geq 0.25 \mathrm{~m}$.

To find the maximum distance that the energy could reach from the transmitter antenna using the Friis equation for the higher frequency part, with the assumption that the minimum accepting energy at that distance is $1000 \mathrm{~W}$, equation (16) should be applied as follows:

$\mathrm{D} \leq \quad+\sqrt[2]{\frac{\mathrm{At} \mathrm{Ar}}{\lambda^{2} * \mathrm{X}} * \mathrm{Pt} * \text { Volume constant }} \leftrightarrow \mathrm{D} \leq$ $+\sqrt[2]{\frac{0.025 * 0.025}{(0.1)^{2} * 1000} *(3 * 10)^{5} * 1}$

So, $\mathrm{D} \leq 4.33 \mathrm{~m}$.

To find the maximum distance (farthest distance could the transmitted energy reach from the transmitter) for the higher frequency part, equation (15) should be applied as follows:

$\mathrm{D} \leq+\sqrt[2]{\frac{\mathrm{At} \mathrm{Ar}}{\lambda^{2}} * \mathrm{Pt} * \text { Volume constant }} \leftrightarrow \quad \mathrm{D} \leq$ $+\sqrt[2]{\frac{0.025 * 0.025}{(0.1)^{2}} *\left(3 * 10^{5}\right) * 1}$

So, $\mathrm{D} \leq 136.93 \mathrm{~m}$.

Then, the theoretical distance range (in meters) for the Friis equation when using $3 \mathrm{GHz}$ frequency with isotropic antenna when using all parameters for the higher frequency part is: $0.25 \leq \mathrm{D} \leq 136.93$.

To find the minimum distance (nearest distance from the transmitter) for higher frequency and directed antenna together part, equation (9) should be applied as follows:

$\mathrm{D} \geq+\sqrt[2]{\frac{\mathrm{AtAr}}{\lambda^{2}} * \text { Volume constant }} \leftrightarrow \quad \mathrm{D} \quad \geq$ $+\sqrt[2]{\frac{0.025 * 0.025}{(0.1)^{2}} * 18334}$

So, $\mathrm{D} \geq 33.85 \mathrm{~m}$.

To find the maximum distance that the energy could reach from the transmitter antenna using the Friis equation for higher frequency and directed antenna together part, with the assumption that the minimum accepting energy at that distance is $1000 \mathrm{~W}$, equation (16) should be applied as follows:

$\mathrm{D} \leq+\sqrt[2]{\frac{\mathrm{At} \mathrm{Ar}}{\lambda^{2} * \mathrm{X}} * \mathrm{Pt} * \text { Volume constant }} \leftrightarrow \quad \mathrm{D} \leq$

$+\sqrt[2]{\frac{0.025 * 0.025}{(0.1)^{2} * 1000} *\left(3 * 10^{5}\right) * 18334}$

So, $\mathrm{D} \leq 586.31 \mathrm{~m}$.

To find the maximum distance (farthest distance could the transmitted energy reach from the transmitter) for higher frequency and directed antenna together part, equation (15) should be applied as follows:

$\mathrm{D} \leq+\sqrt[2]{\frac{\mathrm{At} \mathrm{Ar}}{\lambda^{2}} * \mathrm{Pt} * \text { Volume constant }} \leftrightarrow \quad \mathrm{D} \leq$
$+\sqrt[2]{\frac{0.025 * 0.025}{(0.1)^{2}} *\left(3 * 10^{5}\right) * 18334}$ So, $\mathrm{D} \leq 18,540 \mathrm{~m}$.

Then, the theoretical distance range (in meters) for the Friis equation when using $3 \mathrm{GHz}$ frequency with extremely direct antenna (Volume constant $=18334$ ) when using all parameters for higher frequency and directed antenna together part is: $33.85 \leq \mathrm{D} \leq 18,540$. See Figure 3, it has illustration regarding Friis equation valid distance range.

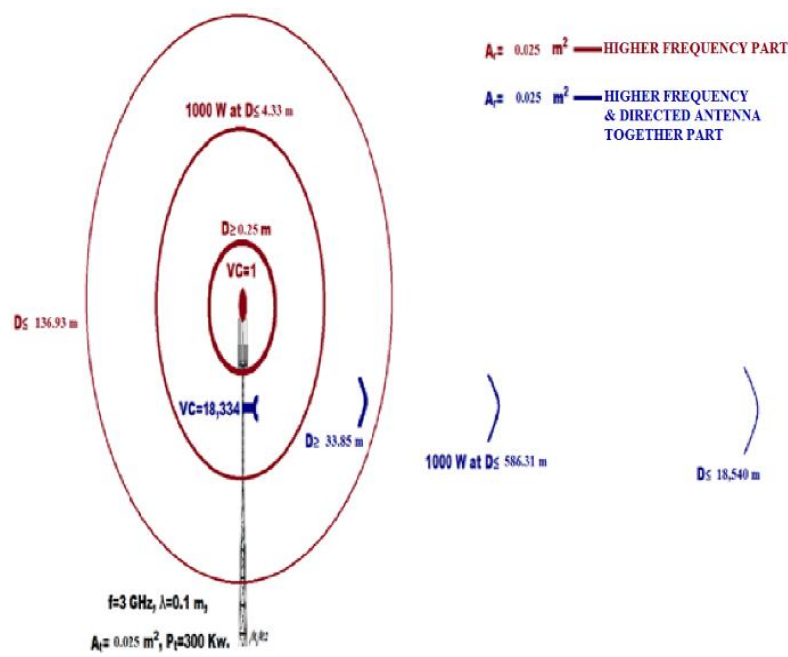

Figure 3: Illustration to Friis equation valid distance range

In fact, there are many factors that can decrease the distance range and the $\mathrm{BE}$, such as [9]: absorption, bad antennas design, diffraction, earth atmosphere layers, polarization rain, reflection (e.g., ground reflecting waves), refraction, scattering.

\section{RESULTS AND DISCUSSION}

There is a difference in the received power between isotropic and directed radiation. The directed radiation is a good solution for the isotropic (undirected) radiation problem. Therefore, Directed radiation is the desired radiation in this research paper (Figure $2(d)$ )

\section{PREDICTED DATA}

In this research paper, MATLAB ${ }^{\circ}$ program is used to find the relations between variables, and to simulate the predicted results (the received power with distance (equation 1), and the beam efficiency with distance (equation 3)) for an isotropic antenna. Using the same frequency (Tesla experiment frequency) $\mathrm{f}=150 \mathrm{kHz}$, wavelength $\lambda=2000 \mathrm{~m}$. Also, keeping At $=60.96 \mathrm{~m} 2$ and $\mathrm{Ar}=60.96 \mathrm{~m} 2\left(\mathrm{At} \leq \frac{1}{2} \lambda, \mathrm{Ar} \leq\right.$ $\frac{1}{2} \lambda$ ). Using transmitting power, $\mathrm{Pt}=5000 \mathrm{~W}$ (300000 is high power for experimental purposes, so 5000 is a preferred value).

The following Figure 4 shows the isotropic antenna's behavior for power received with distance, and the beam efficiency with distance. As shown below, most of the power is wasted. 


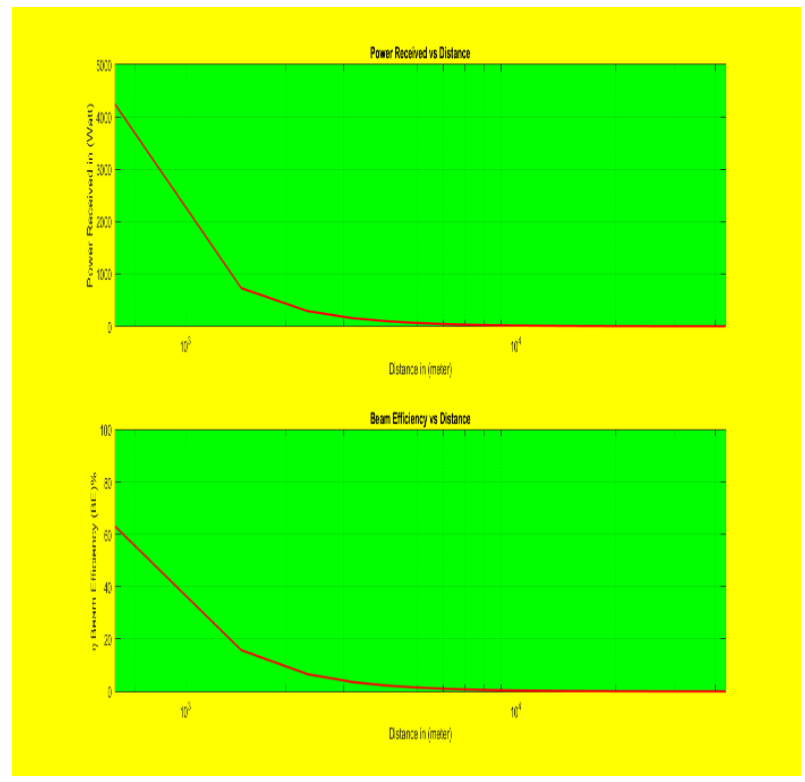

Figure 4: Power received and beam efficiency with distance for an isotropic antenna

\section{DIRECTED ANTENNA APPROACH}

When using a directed antenna that radiates in a specific direction, better results will be achieved compared to an isotropic antenna. The MATLAB $®$ code simulates all parts of Figure 2, and shows the relations between the received power with distance and the beam efficiency with distance for each desired volume (isotropic (full sphere), hemisphere, quarter sphere, and segment). Where PD: is the power density, assuming $\mathrm{PD}=\mathrm{Pr}$, and $\mathrm{Pin}$ is the transmitted power $(\mathrm{Pin}=\mathrm{Pt}$ $=5000 \mathrm{~W}$ ) with the following modification: $4 * \mathrm{R} 2=4 * \mathrm{D} 2$ $=(\lambda * \mathrm{D}) 2$, then $\lambda 2=4, \lambda=2$, then $\mathrm{f}=\mathrm{c} / \lambda=3 * 108 / 2=150$ MHz. Choosing At $=0.75 \mathrm{~m} 2$ and $\mathrm{Ar}=0.75 \mathrm{~m} 2\left(\mathrm{At} \leq \frac{1}{2} \lambda\right.$, Ar $\leq \frac{1}{2} \lambda$ ).

The following Figures $(5,6)$ show the received power for each desired volume with distance, the comparison of the received power of different volumes with distance, the beam efficiency for each desired volume with distance, and the comparison of different volume's beam efficiency with distance respectively.

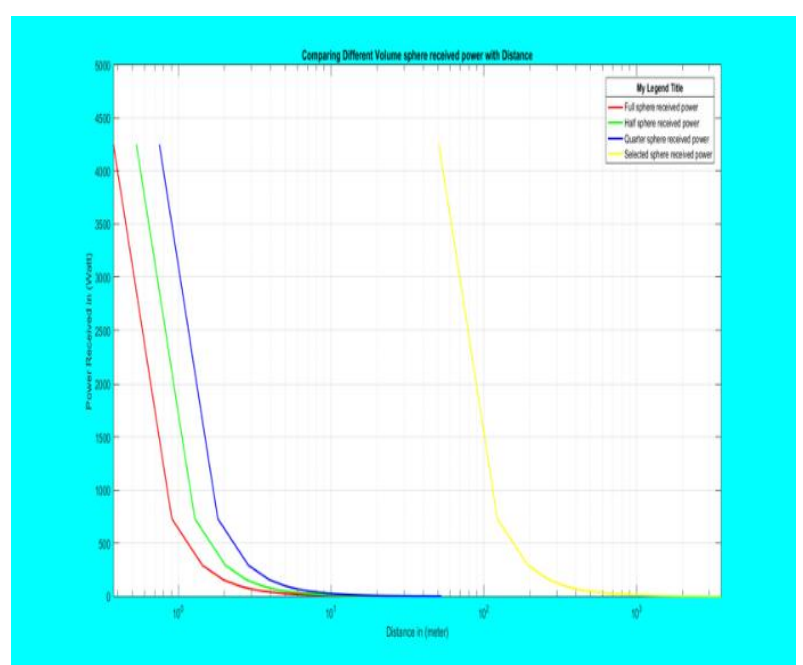

Figure 5: The comparison of the received power of different volumes with distance

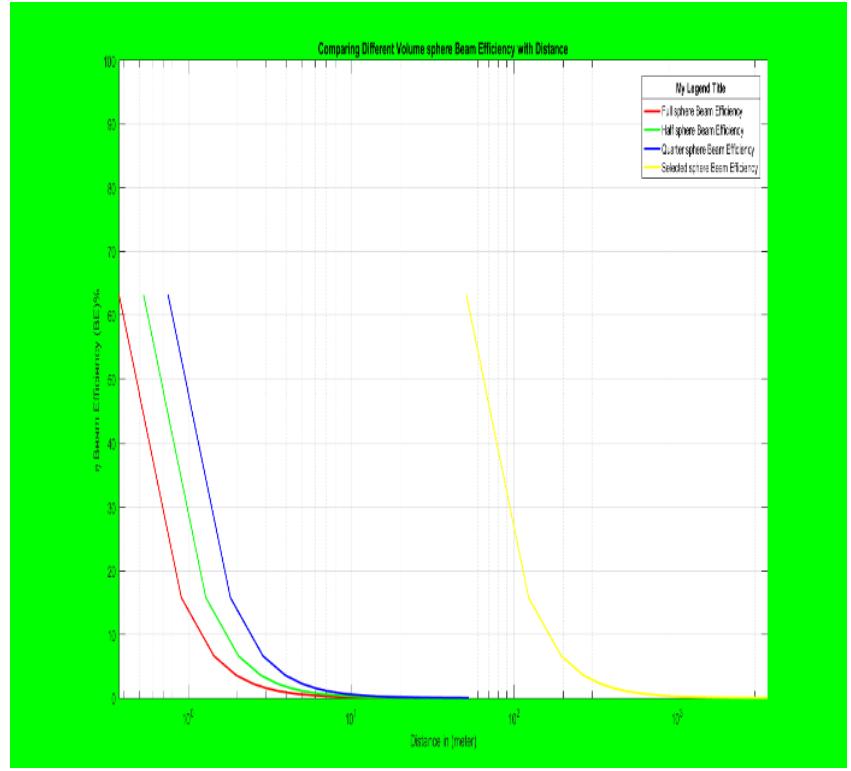

Figure 6: The comparison of different volume's beam efficiency with distance, respectively

\section{CONCLUSION AND FUTURE WORK}

The distance range for (isotropic, hemisphere, quarter sphere) antennas was between 0.25 - 136.93 meters, where the distance range for the directed antenna was $33.85-18,540$ meters. By taking an overall view on both far-field WPT radiated techniques isotropic and directed antenna (such as parabolic antenna), it is safe to conclude that the directed WPT calculations are more convenient in terms of the distance covered. Many parameters can increase the BE, such as the antenna directivity, antenna aperture, antenna size, used frequency, power transmitted, distance, etc. Moreover, even the focused beam MPT could increase the WPT directed antenna's overall efficiency, where the efficient point-to-point power transfer depends on focusing the electromagnetic energy into a narrow beam [3]. Practically, this could be done by using optical lenses or reflectors with very short electromagnetic wavelengths. This research paper aims towards improving the WPT techniques in order to charge electronic devices wirelessly. In addition, isotropic and direct WPT concepts are implemented by using MATLAB ${ }^{\circledR}$ program to show how direct WPT can improve the overall WPT system. The transferred wireless power amount should be enough to meet the minimum requirements to operate some electronic devices. These devices include mobile phones, Portable Computers laptops (PCs), Integrated circuits (ICs), Light Emitting Diodes (LEDs), and even Electric Vehicles (EVs).

In the future, the hardware of the WPT systems can be built, and measure the efficiency along with other performance parameters. Moreover, new methods to improve the efficiency and power density can be investigated. The simulation results verified the feasibility of improving the overall effectiveness (both the received power and the Beam Efficiency (BE)) using directed WPT radiation pattern compared with the isotropic WPT radiation. Future research should consider calculations that use narrower transmitted beam to enhance the proficiency and the productivity of the WPT system. 


\section{REFERENCES}

[1] Schilling, D.R. 2017. WiTricity Charges Forward with Wireless Electricity Available from: http://www.industrytap.com/witricity-charges-forwardwith-wireless-electricity/574.

[2] Liu, C., et al., 2014. Design and Safety Considerations of an Implantable Rectenna for Far-Field Wireless Power Transfer. Antennas and Propagation, IEEE Transactions on, 2014. 62(11): p. 5798-5806.

[3] Brown, W.C. 1984. The History of Power Transmission by Radio Waves. Microwave Theory and Techniques, IEEE Transactions on, 1984. 32(9): p. 1230-1242.

[4] Shinohara, N. 2014 Wireless Power Transfer Via Radiowaves.
[5] Tomar, A. and S. Gupta. 2012. Wireless power transmission: Applications and components. International Journal of Engineering, 2012. 1(5).

[6] Strassner, B. and K. Chang. 2013. Microwave Power Transmission: Historical Milestones and System Components. Proceedings of the IEEE, 2013. 101(6): p. 1379-1396

[7] Shaw, J.A. and Shaw. 2013. Radiometry and the Friis Transmission Equation. American Journal of Physics, 2013. 81(1): p. 33

[8] Antenna Introduction/Basics. 2017. Antenna fundamentals]. Available from: www.phys.hawaii.edu/ anita/new/papers/militaryHandb ook/antennas.pdf. 